\title{
Hypoalbuminemia - An Indicator of the Severity and Prognosis of COVID-19 Patients: A Multicentre Retrospective Analysis
}

\author{
Chaoyue Chen $\mathbb{D}^{1, *}$ \\ Ying Zhang $\mathbb{D}^{1, *}$ \\ Xi Zhao (D) \\ Meihui Tao $\mathbb{D}^{\prime}$ \\ Wei Yan $\mathbb{D}^{2}$ \\ Yu Fu (D)
}

'Department of Gastroenterology, Union Hospital, Tongji Medical College, Huazhong University of Science and Technology, Wuhan, 430022, People's Republic of China; ${ }^{2}$ Department of Gastroenterology, Tongji Hospital, Tongji Medical College, Huazhong University of Science and Technology, Wuhan, 430030, People's Republic of China

*These authors contributed equally to this work
Correspondence: Yu Fu

Department of Gastroenterology, Union Hospital, Tongji Medical College, Huazhong University of Science and Technology, 1277 Jiefang Avenue, Wuhan, 430022, Hubei, People's Republic of China Tel $+86 / 3907 / 94622$

Fax + 8627-8572630

Email futureyu@hust.edu.cn
Background: Hypoalbuminemia has been reported in COVID-19 patients. Exploring the influencing factors and possible adverse consequences of albumin reduction may provide some guidance for the treatment of COVID-19 patients.

Methods: In this multicentre retrospective study, we collected information including demographics, comorbidities, clinical symptoms, complications, laboratory tests, treatment, and outcomes of patients diagnosed with COVID-19 from three hospitals in Wuhan, China. We compared the indexes between patients with hypoalbuminemia and normal albumin. Regression model was used to evaluate various influencing factors of patients with hypoalbuminemia and their relationship with clinical outcomes. We also compared the changes of particular laboratory indexes in patients with hypoalbuminemia before and after enteral nutrition therapy.

Results: A total of 482 patients were enrolled in the study. About 53.7\% patients developed hypoalbuminemia during admission. Patients with hypoalbuminemia were older, had a higher proportion of combined diabetes mellitus, fever, dyspnea, and natriuresis, and had a relatively poorer prognosis than patients with normal albumin. Patients with hypoalbuminemia had higher levels of CRP, leukocytes, ALT, AST, total bilirubin, ALP, GGT, LDH, creatine kinase, D-dimer, globulin, and lower levels of lymphocytes and eosinophils. Severe, older, anorexia, elevated CRP, and decreased lymphocytes were the independent predictors for decreased albumin in COVID-19 patients. In addition, decreased albumin is correlated with adverse outcomes. Nutritional support therapy to correct serum albumin may improve patient outcomes.

Conclusion: COVID-19 patients with hypoalbuminemia tend to have more severe clinical manifestations and more abnormal biochemical tests, which may result in poorer clinical outcomes. Nutritional support therapy may improve the clinical outcome of these patients.

Keywords: hypoalbuminemia, albumin, COVID-19, enteral nutrition therapy

\section{Introduction}

The coronavirus disease 2019 (COVID-19) was first identified in December 2019, in Wuhan, Hubei Province, China and has evolved into a pandemic rapidly. ${ }^{1}$ Based on the World Health Organization, the total number of confirmed cases of COVID19 has exceeded 200 million worldwide by August 5, 2021, with more than 4 million deaths, which is significantly higher than the number of cases and deaths caused by severe acute respiratory syndrome (SARS) in 2003 and has aroused extensive attention among people around the world. 
Even though people have been struggling with SARSCoV-2 virus for more than 18 months, problems such as rare clinical features and effective therapeutic methods still need to be explored.

A previous study included 663 COVID-19 patients showed that $67.0 \%$ patients had lower albumin levels, and reduced albumin was associated with the aggravation of COVID-19. ${ }^{2}$ Patients with in-hospital deaths have lower albumin levels than those without in-hospital deaths. ${ }^{3}$ A growing number of studies have been reporting the presence of hypoproteinemia in COVID-19 patients. Serum albumin is a biochemical marker which is routinely tested in admitted patients and reflects the nutritional status of patients, correlates with liver function, disease morbidity and mortality. Albumin is a negative acute phase protein, which has been found decreased during the acute disease state of COVID-19 disease. ${ }^{4}$ It plays an essential role in controlling plasma osmotic pressure, vascular permeability and transporting various compounds (such as cholesterol, bile pigments, metals, nitric oxide and other molecules). ${ }^{5}$ There are few studies that systematically describe the clinical characteristics and poor prognosis of COVID-19 patients with hypoalbuminemia.

In this retrospective study, we aimed to compare the demographic, clinical, laboratory tests, and imaging characteristics as well as the treatment and outcome of COVID-19 patients with or without hypoalbuminemia during hospitalization, to investigate the effect of albumin reduction on the prognosis of COVID-19, and to explore the possible adverse outcomes caused by hypoalbuminemia. We hope that this study could provide some references for the treatment of COVID-19 patients with hypoalbuminemia.

\section{Methods}

\section{Research Design}

In this multicentre, retrospective and record-based study, data on all adult 482 patients with laboratory-confirmed COVID-19 were collected from Wuhan Tongji Hospital, Union Hospital main district, and Jin Yin-tan Hospital (Wuhan, China) who died or were discharged between February 1, 2020 and February 20, 2020. The main inclusion criteria were the following: confirmed cases of COVID-19 based on the examination of swab specimens by polymerase chain reaction (PCR); patients with serum albumin level drawn on the day of admission. In order to minimize the confounding factors that may affect our assessment of the impact of serum albumin, we excluded patients who were known to have a history of end-stage liver disease (ESLD), cirrhosis, or nephrotic syndrome since these medical conditions may independently alter albumin levels. ${ }^{6}$

The diagnostic criteria and clinical classification criteria of COVID-19 were determined according to the "Diagnosis and Treatment Protocol for Novel Coronavirus Pneumonia (7th interim edition)" issued by the National Health Commission of China. ${ }^{6}$ Four criteria must be met before discharge, including normal body temperature for more than 3 days, significant improvement in respiratory symptoms, significant improvement in pulmonary imaging, and 2 consecutive negative results of throat swabs (at least 24 hours apart).

\section{Data Collection}

We collected 487 patients discharged from 3 hospitals from February 1, 2020 to February 20, 2020, excluding 2 cases with severe data gaps and 3 cases with a history of ESLD, finally included 482 patients for analysis. The records accessed included the patient treatment cards and laboratory registers maintained at the centres from the electronic medical records system through a standardized data collection form. The extracted variables included demographic information, comorbidities (cardiovascular disease, chronic pulmonary disease, diabetes, cancer, autoimmune disease), symptoms (fever, chills, cough, chest distress or pain, dyspnea, fatigue, poor appetite, nausea, vomiting, diarrhea), laboratory tests (complete blood cell count, serum biochemistry test, coagulation profile, liver and renal function, C-reactive protein[CRP], lactate dehydrogenase[LDH], and creatine kinase), imaging examination, complications, and treatment (antivirals agents, antibiotics, glucocorticoids, and mechanical ventilation). Different centres employed uniform lab methods. 10 patients developed hypoalbuminemia during admission and treated with enteral nutrition therapy were selected, recorded the mean value of laboratory tests (albumin, leukocytes, absolute lymphocytes, absolute eosinophils, CRP, and LDH) before and after nutrition therapy for 3 days.

All authors reviewed the extracted data and immediately revised errors by checking the original case data after the errors were found to ensure the accuracy and completeness of the data.

The degrees of disease were classified according to the Diagnosis and Treatment Protocol for Novel Coronavirus 
Pneumonia ( 7 th interim edition) ${ }^{6}$ as mild, normal, severe and critical. In this study, nonsevere including mild and normal, severe including severe and critical. The normal range of serum albumin was $35-55 \mathrm{~g} / \mathrm{L}$, and the standard definition of hypoalbuminemia was albumin $<35 \mathrm{~g} / \mathrm{L}$. The normal range of lymphocyte count was $(1-4) \times 10^{9} / \mathrm{L}$, and lymphopenia was defined as lymphocyte count $<1 \times 10^{9} / \mathrm{L}$. Patients were divided into the hypoalbuminemia group and normal albumin group according to the lowest albumin value during admission was $<35 \mathrm{~g} / \mathrm{L}$ or not. All laboratory parameters, except albumin, were taken from the first laboratory tests on admission.

\section{Statistical Analysis}

Continuous variables were expressed as median and interquartile ranges (IQRs), and categorical variables were described as counts or percentages (\%). Based on the type of distribution of the data, independent $t$-test or MannWhitney $U$-test were performed to analyze continuous variables based on normal or nonnormal distributed data and $\chi^{2}$ test or Fisher's exact test were performed to analyze categorical variables. We divided patients into normal albumin group and hypoalbuminemia group to compare the differences in basic characteristics, laboratory parameters, treatment and other variables between the two groups. Univariate and multifactorial logistic regression methods were used to investigate the factors associated with the adverse outcome of COVID-19 patients with hypoalbuminemia, severe, abnormal liver function, and death, and the odds ratio (OR) was given with 95\% confidence intervals (CIs). After excluding death cases, the factors that influenced the days in hospital and the days from admission to appearing hypoalbuminemia in COVID-19 patients were explored using one-dimensional and multiple linear regression models, and the partial regression coefficients $\beta$ and $95 \%$ CI of each independent variable were recorded. Statistical analysis was performed in SPSS version 22.0. GraphPad Prism 8.0 was used to draw the box diagram of patients' laboratory tests before and after enteral nutrition therapy. Statistical tests were two-tailed. $\mathrm{P}<0.05$ was identified as statistically significant.

\section{Results}

\section{Baseline Characteristics of COVID-19 Patients}

A total of 482 laboratory-confirmed COVID-19 patients were enrolled in this study (Table 1). The median age was 56 (IQR, $39-67)$ years and $243(50.4 \%)$ cases were male. There were
259 (53.7\%) patients with hypoalbuminemia (albumin $<35 \mathrm{~g} /$ $\mathrm{L})$, most of whom were male $(\mathrm{P}=0.006)$ and older $(\mathrm{P}<0.001)$, and the difference was statistically significant. The proportion of severe patients in the hypoalbuminemia group was significantly higher than that in the normal albumin group (58.3\% vs $16.6 \%, \mathrm{p}<0.001)$. Common comorbidities were hypertension (25.3\%) and diabetes mellitus (14.9\%), and a minority of patients had coronary heart disease $(7.7 \%)$, chronic obstructive pulmonary disease $(3.7 \%)$, malignancy $(3.1 \%)$, and autoimmune diseases (3.1\%). A higher proportion of diabetes was present in patients with hypoalbuminemia. Compared with the normal albumin group, a higher proportion of patients in the hypoalbuminemia group had fever $(93.4 \%, \mathrm{p}<0.001)$, dyspnea $(21.6 \%, \mathrm{p}=0.004)$ and myalgia $(52.5 \%, \mathrm{p}<0.001)$, as well as acute respiratory distress syndrome $(16.5 \%, \mathrm{p}<0.001)$, all of which were statistically significant. Patients in the hypoalbuminemia group were more likely to be treated with corticosteroid (54.62\% vs $16.67 \%, \mathrm{p}<0.001)$ and antibiotics $(93.08 \%$ vs $87.39 \%, \mathrm{p}=0.034)$. Patients with hypoalbuminemia had a poorer prognosis and a significantly higher proportion of deaths $(23.85 \%$ vs $0.9 \%, \mathrm{P}<0.001)$ than patients with normal albumin. The proportions of patients in the hypoalbuminemia group who had improved chest CT $(62.7 \%$ vs $75.2 \%, \mathrm{P}<$ $0.001)$, normalized CRP $(28.46 \%$ vs $50.9 \%, \mathrm{P}<0.001)$, and normalized lymphocytes ( $50 \%$ vs $93.69 \%, \mathrm{P}<0.001)$ before hospital discharge were lower than those of patients with normal albumin, which were statistically significant. (Table 1).

\section{Laboratory Findings of COVID-19 Patients}

Among 482 patients, 259 (53.7\%) had serum albumin level of less than $35 \mathrm{~g} / \mathrm{L}$, with a median albumin level of $30.8 \mathrm{~g} / \mathrm{L}$ (IQR 28.5-32.9). In the hypoalbuminemia group, the patients had higher levels of CRP (4.6 vs 42.3, P $<0.001$ ), leukocytes (4.9 vs 5.6, $\mathrm{P}<0.001$ ), lower levels of lymphocytes (1.37 vs $0.86, \mathrm{P}<0.001$ ) and a greater proportion of abnormal CRP, WBCs and lymphocytes than patients with normal albumin. AST $(p<0.001)$, total bilirubin $(p<0.001)$, ALP $(p=0.013)$, GGT $(\mathrm{p}<0.001)$, LDH $(\mathrm{p}<0.001)$, creatine kinase $(\mathrm{p}<0.001)$, D-dimer $(\mathrm{p}<0.001)$, and globulin $(\mathrm{p}<0.001)$ levels were also higher in the hypoalbuminemia group than the normal albumin group (Table 2).

\section{Factors Influencing the Decrease in Albumin}

Multifactorial logistic regression results showed (Table 3) that severe (OR 2.654, [95\% CI, 1.528- 
Table I Differences in COVID-19 Patients Characteristics Between Hypoalbuminemia and Normal Albumin Group

\begin{tabular}{|c|c|c|c|c|}
\hline \multirow[t]{2}{*}{ Indicators } & \multirow[t]{2}{*}{ Total $(n=482)$} & \multicolumn{2}{|c|}{ Albumin } & \multirow[t]{2}{*}{$P$ value } \\
\hline & & Albumin $\geq 35 \quad(n=223,46.3 \%)$ & Albumin $<35(n=259,53.7 \%)$ & \\
\hline Age, median (IQR) & $56(39-67)$ & $44(34-63)$ & 64(49-69) & $<0.001$ \\
\hline \multicolumn{5}{|l|}{ Sex } \\
\hline Male & $243(50.4 \%)$ & $97(43.5 \%)$ & $146(56.4 \%)$ & 0.0060 \\
\hline Female & $239(49.6 \%)$ & $126(56.5 \%)$ & $113(43.6 \%)$ & \\
\hline \multicolumn{5}{|l|}{ Severity } \\
\hline Nonsevere & $294(61.0 \%)$ & $186(83.4 \%)$ & $108(41.7 \%)$ & $<0.001$ \\
\hline Severe & $188(39.0 \%)$ & $37(16.6 \%)$ & $15 \mathrm{I}(58.3 \%)$ & \\
\hline Hospital stays, median (IQR) days & $13(10-17)$ & $13(10-16)$ & $13(10-18)$ & 0.3000 \\
\hline \multicolumn{5}{|l|}{ Comorbidities } \\
\hline Hypertension & $122(25.3 \%)$ & $49(22.1 \%)$ & $73(28.1 \%)$ & 0.1310 \\
\hline Diabetes & $72(14.9 \%)$ & $24(10.8 \%)$ & $48(18.5 \%)$ & 0.0190 \\
\hline Cardiovascular disease & $37(7.7 \%)$ & $13(5.9 \%)$ & $24(9.2 \%)$ & 0.1650 \\
\hline COPD & $18(3.7 \%)$ & $8(3.60 \%)$ & $10(3.9 \%)$ & 0.889 \\
\hline Malignancy & $15(3.1 \%)$ & $4(1.80 \%)$ & II (4.2\%) & 0.1260 \\
\hline Autoimmune diseases & $15(3.1 \%)$ & $7(3.17 \%)$ & $8(3.1 \%)$ & 0.9610 \\
\hline \multicolumn{5}{|l|}{ Signs and symptoms } \\
\hline Fever & $424(88.0 \%)$ & $182(81.6 \%)$ & $242(93.4 \%)$ & $<0.001$ \\
\hline Rigor & $58(12.0 \%)$ & $22(9.9 \%)$ & $36(13.9 \%)$ & 0.1750 \\
\hline Cough & $289(60.0 \%)$ & $130(58.3 \%)$ & $159(6 \mid .4 \%)$ & 0.4890 \\
\hline Chest distress or pain & $145(30.1)$ & $69(30.9 \%)$ & $76(29.3 \%)$ & 0.7030 \\
\hline Dyspnea & $82(17.0 \%)$ & $26(11.7 \%)$ & $56(21.6 \%)$ & 0.0040 \\
\hline Fatigue & 159(33.0\%) & $69(30.9 \%)$ & $90(34.8 \%)$ & 0.4110 \\
\hline Myalgia & $193(40.0 \%)$ & $57(25.6 \%)$ & $136(52.5 \%)$ & $<0.001$ \\
\hline Nausea & $28(5.8 \%)$ & $9(4.1 \%)$ & $19(7.3 \%)$ & 0.1280 \\
\hline Vomiting & $27(5.6 \%)$ & $10(4.5 \%)$ & $17(6.6 \%)$ & 0.3340 \\
\hline Diarrhea & $129(26.8 \%)$ & $54(24.2 \%)$ & $75(29.0 \%)$ & 0.2410 \\
\hline \multicolumn{5}{|l|}{ Complications } \\
\hline ARDS & $44(9.1 \%)$ & $\mathrm{I}(0.5 \%)$ & $43(16.5 \%)$ & $<0.001$ \\
\hline Acute kidney injury & $47(9.8 \%)$ & $16(7.2 \%)$ & $31(11.9 \%)$ & 0.0820 \\
\hline Acute cardiac injury & $7(1.5 \%)$ & $3(1.4 \%)$ & $4(1.5 \%)$ & 1.0000 \\
\hline DIC & $\mathrm{I}(0.2 \%)$ & $0(0 \%)$ & $\mathrm{I}(0.4 \%)$ & 1.0000 \\
\hline \multicolumn{5}{|l|}{ Pulmonary CT presentation on admission } \\
\hline With signs of viral pneumonia & $475(98.5 \%)$ & $217(97.3 \%)$ & $257(99.2 \%)$ & 0.2560 \\
\hline No obvious abnormalities & $7(1.5 \%)$ & $6(3.7 \%)$ & $2(0.8 \%)$ & \\
\hline \multicolumn{5}{|l|}{ Discharge status } \\
\hline Chest CT normal or fibrosis & $49(10.2 \%)$ & $40(18.0 \%)$ & $9(3.5 \%)$ & $<0.001$ \\
\hline Chest CT improvement & $330(68.8 \%)$ & $167(75.2 \%)$ & $163(62.7 \%)$ & $<0.001$ \\
\hline CRP normal & $187(38.8 \%)$ & $113(50.9 \%)$ & $74(28.46 \%)$ & $<0.001$ \\
\hline Lymphocyte count normal & $338(70.1 \%)$ & $208(93.69 \%)$ & $130(50 \%)$ & $<0.001$ \\
\hline \multicolumn{5}{|l|}{ Treatment after admission } \\
\hline Antiviral & $472(97.9 \%)$ & $215(96.85 \%)$ & $257(98.85 \%)$ & 0.1980 \\
\hline Antibiotic & $436(90.5 \%)$ & 194(87.39\%) & $242(93.08 \%)$ & 0.0340 \\
\hline Corticosteroid & $179(37.1 \%)$ & $37(16.67 \%)$ & $142(54.62 \%)$ & $<0.001$ \\
\hline Mechanical ventilation & $66(13.7 \%)$ & $26(11.71 \%)$ & $40(15.38 \%)$ & 0.2420 \\
\hline
\end{tabular}


Table I (Continued).

\begin{tabular}{|c|c|c|c|c|}
\hline \multirow[t]{2}{*}{ Indicators } & \multirow[t]{2}{*}{ Total $(n=482)$} & \multicolumn{2}{|c|}{ Albumin } & \multirow[t]{2}{*}{$P$ value } \\
\hline & & Albumin $\geq 35(n=223,46.3 \%)$ & Albumin $<35(n=259,53.7 \%)$ & \\
\hline \multicolumn{5}{|c|}{ Clinical outcome } \\
\hline Discharged & $418(86.7 \%)$ & $221(99.10 \%)$ & $197(76.15 \%)$ & $<0.001$ \\
\hline Died & $64(13.3 \%)$ & $2(0.9 \%)$ & $62(23.85 \%)$ & \\
\hline
\end{tabular}

Abbreviations: COVID-19, coronavirus disease 2019; IQR, interquartile range; COPD, chronic obstructive pulmonary disease; ARDS, acute respiratory distress syndrome; DIC, diffuse intravascular coagulation; CRP, C-reactive protein.

Table 2 Laboratory Findings in COVID-I9 Patients Characteristics Between Hypoalbuminemia and Normal Albumin Group

\begin{tabular}{|c|c|c|c|c|}
\hline \multirow[t]{2}{*}{ Indicators } & \multirow[t]{2}{*}{ Total $(n=482)$} & \multicolumn{2}{|c|}{ Albumin } & \multirow[t]{2}{*}{$P$ value } \\
\hline & & $\geq 35(223,42.3 \%)$ & $<35(259,53.7 \%)$ & \\
\hline Albumin, $g / L$ & $34.15(30.6-38.0)$ & $38.3(36.6-39.7)$ & $30.8(28.5-32.9)$ & \\
\hline CRP, mg/L & $16.1(3.5-5 \mid .3)$ & $4.6(0.8-14.5)$ & $42.3(0.5-9.1)$ & $<0.001$ \\
\hline$>10$ & $268 / 46 \mid(58.1 \%)$ & 69/209(33.0\%) & 199/252(79.0\%) & $<0.001$ \\
\hline WBC count, $\times 10^{9} / \mathrm{L}$ & $5.2(3.9-6.9)$ & $4.9(3.8-6.2)$ & $5.6(4.0-7.6)$ & $<0.001$ \\
\hline$<4$ & $|29 / 48|(26.8 \%)$ & $65 / 222(29.3 \%)$ & $64 / 259(24.7 \%)$ & NA \\
\hline $4-10$ & $3|4 / 48|(65.3 \%)$ & $156 / 222(70.3 \%)$ & $158 / 259(61.0 \%)$ & NA \\
\hline$>10$ & $38 / 48 I(7.9 \%)$ & $\mathrm{I} / 222(0.5 \%)$ & $37 / 259(14.3 \%)$ & $<0.001$ \\
\hline Platelet count, $\times 10^{9} / \mathrm{L}$ & $202(149-265)$ & $206(153.3-266.8)$ & $194.5(\mid 40.7-258.3)$ & $0.07 \mid$ \\
\hline Lymphocyte count, $\times 10^{9} / \mathrm{L}$ & I.I (0.73-I.50) & $1.37(1.03-1.78)$ & $0.86(0.64-1.24)$ & $<0.001$ \\
\hline$<1.0$ & $202 / 48 I(42.0 \%)$ & $48 / 222(21.6 \%)$ & $154 / 259(59.4 \%)$ & $<0.001$ \\
\hline Eosinophil count, $\times 10^{9} / \mathrm{L}$ & $0.02(0-0.07)$ & $0.04(0.01-0.09)$ & $0.01(0-0.06)$ & $<0.001$ \\
\hline$<0.02$ & $226 / 48 I(47.0 \%)$ & $72 / 222(32.4 \%)$ & $154 / 259(59.5 \%)$ & $<0.001$ \\
\hline Creatinine, $\mu \mathrm{mol} / \mathrm{L}$ & $68(57.0-83.7)$ & $66.1(57.4-79.8)$ & $70(56.3-86)$ & 0.074 \\
\hline$>133$ & $15 / 479(3.1 \%)$ & $3 / 222(1.4 \%)$ & $12 / 257(4.7 \%)$ & 0.069 \\
\hline ALT, U/L & $21(15-35)$ & $19(13.5-28)$ & $24(17-42.3)$ & $<0.001$ \\
\hline$>40$ & $96 / 482(19.9 \%)$ & $25 / 223(11.2 \%)$ & $71 / 259(27.4 \%)$ & $<0.001$ \\
\hline AST, U/L & $26(19-37)$ & $22(17-28.5)$ & $32(23-46)$ & $<0.001$ \\
\hline$>40$ & $98 / 482(20.3 \%)$ & $17 / 223(7.6 \%)$ & $81 / 259(31.3 \%)$ & $<0.001$ \\
\hline TBil, $\mu \mathrm{mol} / \mathrm{L}$ & $9.1(6.6-12.6)$ & $8(6-10.4)$ & $10.4(7.6-13.6)$ & $<0.001$ \\
\hline ALP, U/L & $64(53-79)$ & $62(52-75)$ & $66(52.7-84)$ & 0.013 \\
\hline$\gamma$-GT, U/L & $26(16-48)$ & $21(14-36.5)$ & $30(19-62.3)$ & $<0.001$ \\
\hline $\mathrm{LDH}, \mathrm{U} / \mathrm{L}$ & $244(|93-33|)$ & $206(177-253)$ & $307.5(235-437)$ & $<0.001$ \\
\hline$>245$ & $223 / 450(49.6 \%)$ & $56 / 209(26.8 \%)$ & $167 / 238(70.2 \%)$ & $<0.001$ \\
\hline $\mathrm{CK}, \mu \mathrm{mol} / \mathrm{L}$ & $70(48-133)$ & $50(3-81.5)$ & $90(51.5-164)$ & $<0.001$ \\
\hline$>185$ & $42 / 262(16.0 \%)$ & $\mathrm{II} / \mathrm{I} 20(9.2 \%)$ & $31 / 142(21.8 \%)$ & 0.005 \\
\hline PT, seconds & $\mid 3.4(|2.7-| 4.2)$ & $13.3(12.8-14)$ & $13.6(\mid 2.3-14.4)$ & 0.156 \\
\hline$\geq 16$ & $24 / 458(5.2 \%)$ & $2 / 210(1.0 \%)$ & $22 / 248(8.9 \%)$ & $<0.001$ \\
\hline APTT, seconds & $37.2(33 .|-4| .3)$ & $38(34.4-4 I .7)$ & $36.3(29.7-40.8)$ & 0.006 \\
\hline D-dimer, $\mu \mathrm{g} / \mathrm{mL}$ & $0.58(0.3|-| .33)$ & $0.36(0.22-0.60)$ & $0.95(0.49-2.2)$ & $<0.001$ \\
\hline$\geq 0.5$ & $244 / 446(54.7 \%)$ & $66 / 204(32.4 \%)$ & I78/242(73.6\%) & $<0.001$ \\
\hline Hemoglobin, g/L & $129(118-138)$ & $129(120-140)$ & $128(116-137)$ & 0.105 \\
\hline Globulin, g/L & $3 I(27.7-34.5)$ & $29.2(26.3-32.1)$ & $32.7(29.4-36.3)$ & $<0.001$ \\
\hline
\end{tabular}

Abbreviations: COVID-19, coronavirus disease 2019; CRP, C-reactive protein; WBC, white blood cell; ALT, alanine aminotransferase; AST, aspartate aminotransferase; TBil, total bilirubin; ALP, alkaline phosphatase; $\gamma$-GT, $\gamma$-glutamyl transpeptidase; LDH, lactate dehydrogenase; CK, Creatine kinase; PT, Prothrombin time; APTT, activated partial thromboplastin time; NA, not available. 
Table 3 Univariate and Multivariate Analysis of COVID-19 Patients with Hypoalbuminemia

\begin{tabular}{|c|c|c|c|c|}
\hline Indicators & Univariate Analysis OR $(95 \% \mathrm{Cl})$ & $P$ value & Multivariate Analysis OR $(95 \% \mathrm{Cl})$ & $P$ value \\
\hline \multicolumn{5}{|l|}{ Baseline characteristics } \\
\hline Age, years & $1.053(1.04-1.067)$ & $<0.001$ & $1.044(1.025-1.062)$ & $<0.001$ \\
\hline Sex, male & $1.622(1.131-2.327)$ & 0.009 & $0.865(0.506-1.477)$ & 0.595 \\
\hline \multicolumn{5}{|l|}{ Severity } \\
\hline Severe & $5.761(3.807-8.716)$ & $<0.001$ & $2.654(1.528-4.607)$ & 0.001 \\
\hline Diabetes & $1.942(1.158-3.255)$ & 0.012 & $0.907(0.44-I .87 I)$ & 0.792 \\
\hline \multicolumn{5}{|l|}{ Signs and symptoms } \\
\hline Fever & $3.207(1.765-5.827)$ & $<0.001$ & $1.908(0.886-4.111)$ & 0.099 \\
\hline Anorexia & $2.182(1.508-3.158)$ & $<0.001$ & $1.93 \mathrm{I}(1.165-3.201)$ & 0.011 \\
\hline Dyspnea & $2.185(1.311-3.64)$ & 0.003 & $0.955(0.458-1.991)$ & 0.902 \\
\hline \multicolumn{5}{|l|}{ Laboratory findings } \\
\hline CRP, mg/L & $1.050(1.039-1.061)$ & $<0.001$ & $1.038(1.024-1.052)$ & $<0.001$ \\
\hline WBC count, $\times 10^{9} / \mathrm{L}$ & $1.204(1.111-1.296)$ & $<0.001$ & $1.005(0.897-1.16)$ & 0.947 \\
\hline Lymphocyte count, $\times 10^{9} / \mathrm{L}$ & $0.144(0.092-0.224)$ & $<0.001$ & $0.55 I(0.303-1.00 I)$ & 0.050 \\
\hline Eosinophil count, $\times 10^{9} / \mathrm{L}$ & $0.004(0-0.008)$ & $<0.001$ & $0.322(0.008-13.598)$ & 0.553 \\
\hline Creatinine, $\mu \mathrm{mol} / \mathrm{L}$ & $1.003(0.998-1.008)$ & 0.177 & NA & NA \\
\hline $\mathrm{ALT}, \mathrm{U} / \mathrm{L}$ & $1.012(1.005-1.019)$ & 0.001 & $1.014(0.997-1.031)$ & 0.108 \\
\hline AST, U/L & $1.044(1.029-1.058)$ & $<0.001$ & $0.984(0.953-1.016)$ & 0.317 \\
\hline Total bilirubin, $\mu \mathrm{mol} / \mathrm{L}$ & $1.095(1.052-1.14)$ & $<0.001$ & $1.012(0.953-1.076)$ & 0.695 \\
\hline ALP, U/L & $1.011(1.005-1.018)$ & 0.001 & $1.004(0.99 \mid-1.018)$ & 0.514 \\
\hline$\gamma-\mathrm{GT}, \mathrm{U} / \mathrm{L}$ & $1.008(1.003-1.013)$ & 0.001 & $0.998(0.992-1.004)$ & 0.599 \\
\hline $\mathrm{LDH}, \mathrm{U} / \mathrm{L}$ & $1.01(1.007-1.012)$ & $<0.001$ & $1.00 I(0.998-1.005)$ & 0.400 \\
\hline $\mathrm{CK}, \mu \mathrm{mol} / \mathrm{L}$ & $1.003(1.002-1.005)$ & $<0.001$ & $0.999(0.997-1.001)$ & 0.328 \\
\hline PT, seconds & $1.003(0.977-1.029)$ & 0.827 & NA & NA \\
\hline APTT, seconds & $0.991(0.971-1.011)$ & 0.352 & NA & NA \\
\hline D-dimer, $\mu \mathrm{g} / \mathrm{mL}$ & $1.098(1.042-1.158)$ & $<0.001$ & $0.972(0.907-1.042)$ & 0.426 \\
\hline Hemoglobin, g/L & $0.996(0.987-1.004)$ & 0.298 & NA & NA \\
\hline Globulin, g/L & $1.152(1.105-1.201)$ & $<0.001$ & $0.999(0.949-1.052)$ & 0.963 \\
\hline
\end{tabular}

Abbreviations: COVID-19, coronavirus disease 2019; OR, odds ratio; Cl, confidence interval; CRP, C-reactive protein; WBC, white blood cell; ALT, alanine aminotransferase; AST, aspartate aminotransferase; TBil, total bilirubin; ALP, alkaline phosphatase; $\gamma$-GT, $\gamma$-glutamyl transpeptidase; LDH, lactate dehydrogenase; CK, Creatine kinase; PT, Prothrombin time; APTT, activated partial thromboplastin time; NA, not available.

4.607]; $\mathrm{p}=0.001$ ), older (OR 1.044, [95\% CI, 1.025$1.062] ; \mathrm{p}<0.001$ ), and anorexia (OR 1.931 [95\% CI, 1.165-3.201]; $\mathrm{P}=0.011$ ), elevated CRP (OR 1.038, [95\% CI (1.024-1.052)], P<0.001), and decreased lymphocytes (OR 0.551 [95\% CI,0.303-1.001], $\mathrm{P}=0.050$ ) were independent predictors in COVID-19 patients with decreased albumin (Table 3).

\section{Outcome Variables That Can Be}

\section{Influenced by Hypoalbuminemia}

Univariate logistic regression showed that hypoalbuminemia was associated with disease severity (OR 5.761, [95\% CI, 3.807-8.716]; $\mathrm{P}<0.001$ ), abnormal liver function (OR 2.760, [95\% CI, 1.884-4.045]; $\mathrm{P}<0.001$ ), and death (OR 34.777, [95\% CI, 8.397-144.036]; $\mathrm{P}<0.001)$. Multifactorial logistic regression showed that hypoalbuminemia was associated with disease severity (OR 2.121, [95\% CI, 1.2583.577]; $\mathrm{P}=0.005$ ). Abnormal liver function ( $\mathrm{OR} 1.434$, [95\% CI, 0.861-2.389]; $\mathrm{P}=0.166$ ), and death (OR 4.012, [95\% CI, 0.317-50.806]; $\mathrm{P}=0.283$ ) lacked correlation with serum albumin (Tables 4-6).

Multiple linear regression analysis suggested a positive correlation between hypoalbuminemia and the days of hospital stays ( $\beta 1.479$ [95\% CI, 0.3-2.658]; $\mathrm{P}=0.014$ ). (Table 7).

\section{Factors Affecting Days Between the Appearance of Hypoalbuminemia and Hospital Admission}

Univariate linear regression analysis suggested that increasing age $(\beta-0.039$ [95\% CI, $-0.066,-0.012]$; 
Table 4 Univariate and Multivariate Analysis of COVID-19 Patients with Severity

\begin{tabular}{|c|c|c|c|c|}
\hline Indicators & Univariate Analysis OR $(95 \% \mathrm{Cl})$ & $P$ value & Multivariate Analysis OR $(95 \% \mathrm{Cl})$ & $P$ value \\
\hline \multicolumn{5}{|l|}{ Baseline characteristics } \\
\hline Age, years & $1.034(1.021-1.046)$ & $<0.001$ & $1.011(0.995-1.027)$ & 0.178 \\
\hline \multicolumn{5}{|l|}{ Signs and symptoms } \\
\hline Anorexia & $1.497(1.037-2.163)$ & 0.031 & $0.936(0.599-1.464)$ & 0.773 \\
\hline Fever & $3.213(1.621-6.369)$ & 0.001 & $1.806(0.831-3.929)$ & 0.136 \\
\hline \multicolumn{5}{|l|}{ Laboratory findings } \\
\hline Hypoalbuminemia & $5.761(3.807-8.716)$ & $<0.001$ & $2.121(1.258-3.577)$ & 0.005 \\
\hline WBC count, $\times 10^{9} / \mathrm{L}$ & $1.258(1.164-1.359)$ & $<0.001$ & $1.17(1.037-1.32)$ & 0.011 \\
\hline Eosinophil count, $\times 10^{9} / \mathrm{L}$ & 0 & $<0.001$ & $0.003(0-0.382)$ & 0.019 \\
\hline Lymphocyte count, $\times 10^{9} / \mathrm{L}$ & $0.171(0.109-0.267)$ & $<0.001$ & $0.432(0.248-0.755)$ & 0.003 \\
\hline CRP, mg/L & $1.019(1.014-1.024)$ & $<0.001$ & $0.999(0.992-1.006)$ & 0.761 \\
\hline AST, U/L & $1.021(1.012-1.031)$ & $<0.001$ & $0.992(0.98-1.004)$ & 0.196 \\
\hline LDH, U/L & $1.008(1.006-1.01)$ & $<0.001$ & $1.004(1.001-1.007)$ & 0.009 \\
\hline D-dimer, $\mu \mathrm{g} / \mathrm{mL}$ & $1.129(1.072-1.19)$ & $<0.001$ & $1.017(0.969-1.067)$ & 0.496 \\
\hline
\end{tabular}

Abbreviations: COVID-19, coronavirus disease 2019; OR, odds ratio; Cl, confidence interval; WBC, white blood cell; CRP, C-reactive protein; AST, aspartate aminotransferase; $\mathrm{LDH}$, lactate dehydrogenase.

Table 5 Univariate and Multivariate Analysis of COVID-19 Patients with Abnormal Liver Biochemistries

\begin{tabular}{|c|c|c|c|c|}
\hline Indicators & Univariate Analysis OR (95\% Cl) & P value & Multivariate Analysis OR $(95 \% \mathrm{Cl})$ & $P$ value \\
\hline \multicolumn{5}{|l|}{ Severity } \\
\hline Severe & $2.266(1.558-3.297)$ & $<0.001$ & $\mathrm{I} .46(0.90 \mathrm{I}-2.364)$ & 0.124 \\
\hline \multicolumn{5}{|l|}{ Complications } \\
\hline Diabetes & I.52(0.929-2.487) & 0.095 & $1.183(0.666-2.102)$ & 0.566 \\
\hline \multicolumn{5}{|l|}{ Signs and symptoms } \\
\hline Fever & $4.838(2.239-10.455)$ & $<0.001$ & $3.075(1.349-7.01 \mathrm{I})$ & 0.008 \\
\hline Anorexia & $1.918(1.325-2.776)$ & 0.001 & $1.732(1.126-2.663)$ & 0.012 \\
\hline Dyspnea & $2.568(1.577-4.183)$ & $<0.001$ & $1.719(0.976-3.03)$ & 0.061 \\
\hline \multicolumn{5}{|l|}{ Laboratory findings } \\
\hline Albumin, g/L & $2.760(1.884-4.045)$ & $<0.001$ & $\mathrm{I} .434(0.86 \mathrm{I}-2.389)$ & 0.166 \\
\hline Globulin, g/L & $1.085(1.046-1.127)$ & $<0.001$ & $1.026(0.98 I-1.073)$ & 0.267 \\
\hline CRP, mg/L & $1.013(1.009-1.018)$ & $<0.001$ & $1.006(0.999-1.012)$ & 0.073 \\
\hline WBC count, $\times 10^{9} / \mathrm{L}$ & $1.066(1.006-1.131)$ & 0.031 & $0.964(0.883-1.053)$ & 0.421 \\
\hline Lymphocyte count, $\times 10^{9} / \mathrm{L}$ & $0.547(0.384-0.779)$ & 0.001 & I.199(0.747-I.925) & $0.45 I$ \\
\hline Eosinophil count, $\times 10^{9} / \mathrm{L}$ & $0.006(0-0.185)$ & 0.003 & $0.089(0.002-3.198)$ & 0.185 \\
\hline ALT, U/L & $1.02(1.012-1.028)$ & $<0.001$ & $1.014(1.001-1.028)$ & 0.036 \\
\hline AST, U/L & $1.03(1.019-1.041)$ & $<0.001$ & $1.002(0.982-1.022)$ & 0.869 \\
\hline ALP, U/L & $1.007(1.001-1.012)$ & 0.017 & $0.997(0.987-1.006)$ & 0.475 \\
\hline$\gamma$-GT, U/L & $1.008(1.003-1.012)$ & $<0.001$ & $1.003(0.998-1.009)$ & 0.211 \\
\hline $\mathrm{LDH}, \mathrm{U} / \mathrm{L}$ & $1.002(1.001-1.003)$ & $<0.001$ & $0.999(0.998-1.001)$ & 0.456 \\
\hline APTT, seconds & $1.034(1.009-1.058)$ & 0.007 & $1.04(1.012-1.07)$ & 0.005 \\
\hline
\end{tabular}

Abbreviations: COVID-19, coronavirus disease 2019; OR, odds ratio; Cl, confidence interval; CRP, C-reactive protein; WBC, white blood cell; ALT, alanine aminotransferase; AST, aspartate aminotransferase; ALP, alkaline phosphatase; $\gamma$-GT, $\gamma$-glutamyl transpeptidase; LDH, lactate dehydrogenase; APTT, activated partial thromboplastin time.

$\mathrm{P}=0.005)$, dyspnea $(\beta-1.182$ [95\% CI, $-2.127,-0.237]$; $\mathrm{P}=0.014)$, elevated CRP $(\beta-0.014 \quad[95 \%$ CI, -0.02 , $-0.007] ; \mathrm{P}<0.001)$ and WBCs $(\beta-0.17$ [95\% CI, -0.27 ,
$-0.069] ; \mathrm{P}=0.001)$ were all negatively associated with the number of days between the patients' appearance of hypoalbuminemia and hospital admission. Multiple linear 
Table 6 Univariate and Multivariate Analysis of COVID-19 Patients with Death

\begin{tabular}{|c|c|c|c|c|}
\hline Indicators & Univariate Analysis OR (95\% Cl) & P value & Multivariate Analysis OR $(95 \% \mathrm{Cl})$ & $P$ value \\
\hline Sex, male & $1.906(1.105-3.289)$ & 0.02 & $0.854(0.225-3.232)$ & 0.816 \\
\hline Age, years & $1.098(1.069-1.128)$ & $<0.001$ & $1.126(1.056-1.2)$ & $<0.001$ \\
\hline Diabetes & $2.938(1.614-5.348)$ & $<0.001$ & $2.444(0.634-9.42)$ & 0.194 \\
\hline Fever & $9.947(1.353-73.143)$ & 0.024 & $\mid 5.352(0.64|-367.5| 2)$ & 0.092 \\
\hline Dyspnea & $4.516(2.544-8.016)$ & $<0.001$ & $3.007(0.835-10.82)$ & 0.092 \\
\hline CRP, mg/L & $1.025(1.019-1.03)$ & $<0.001$ & $0.996(0.983-1.009)$ & 0.557 \\
\hline WBC count, $\times 10^{9} / \mathrm{L}$ & $1.602(|.43|-1.794)$ & $<0.001$ & $1.373(1.097-1.72)$ & 0.006 \\
\hline Lymphocyte count, $\times 10^{9} / \mathrm{L}$ & $0.024(0.009-0.063)$ & $<0.001$ & $0.321(0.056-1.827)$ & 0.2 \\
\hline Eosinophil count, $\times 10^{9} / \mathrm{L}$ & $0(0-0)$ & $<0.001$ & $0(0-0.004)$ & 0.016 \\
\hline Albumin, $g / L$ & $34.777(8.397-144.036)$ & $<0.001$ & $4.012(0.317-50.806)$ & 0.283 \\
\hline Globulin, g/L & 1.175 (1.109-1.244) & $<0.001$ & $0.944(0.817-1.089)$ & 0.427 \\
\hline AST, U/L & $1.024(1.015-1.034)$ & $<0.001$ & $0.98(0.952-1.009)$ & 0.171 \\
\hline Creatinine, $\mu \mathrm{mol} / \mathrm{L}$ & $1.007(1.002-1.013)$ & 0.01 & $1.006(0.997-1.016)$ & 0.207 \\
\hline TBil, $\mu \mathrm{mol} / \mathrm{L}$ & $1.146(1.095-1.199)$ & $<0.001$ & $1.028(0.9101 .161)$ & 0.657 \\
\hline ALP, U/L & $1.009(1.003-1.015)$ & 0.004 & I (0.98-1.02) & 0.997 \\
\hline $\mathrm{LDH}, \mathrm{U} / \mathrm{L}$ & $1.013(1.01-1.015)$ & $<0.001$ & $1.011(1.005-1.017)$ & 0.001 \\
\hline $\mathrm{CK}, \mu \mathrm{mol} / \mathrm{L}$ & $1.003(1.002-1.005)$ & $<0.001$ & I (0.996-I.003) & 0.953 \\
\hline APTT, seconds & $1.054(1.018-1.091)$ & 0.003 & $1.14(1.044-1.244)$ & 0.003 \\
\hline D-dimer, $\mu g / m L$ & $1.2(1.143-1.26)$ & $<0.001$ & $1.057(0.978-1.142)$ & 0.161 \\
\hline
\end{tabular}

Abbreviations: COVID-19, coronavirus disease 2019; OR, odds ratio; Cl, confidence interval; CRP, C-reactive protein; WBC, white blood cell; AST, aspartate aminotransferase; TBil, total bilirubin; ALP, alkaline phosphatase; LDH, lactate dehydrogenase; CK, Creatine kinase; APTT, activated partial thromboplastin time.

Table 7 Linear Regression Analysis of Hospital Stays for Patients with COVID-19

\begin{tabular}{|c|c|c|c|c|c|c|}
\hline \multirow[t]{2}{*}{ Indicators } & \multicolumn{2}{|c|}{ Univariate Linear Regression } & \multirow[t]{2}{*}{$\mathbf{P}$} & \multicolumn{2}{|c|}{ Multiple Linear Regression } & \multirow[t]{2}{*}{$\mathbf{P}$} \\
\hline & $\boldsymbol{\beta}$ & $95 \% \mathrm{Cl}$ & & $\beta$ & $95 \% \mathrm{Cl}$ & \\
\hline Age, years & -0.004 & $(-0.038,0.03)$ & 0.828 & NA & NA & NA \\
\hline Anorexia & 0.751 & $(-0.325,1.827)$ & 0.171 & NA & NA & NA \\
\hline Fever & 1.429 & $(-0.116,2.973)$ & 0.070 & NA & NA & NA \\
\hline Severe & 2.647 & $(1.518,3.777)$ & $<0.001$ & 1.84 & $(0.686,2.995)$ & 0.002 \\
\hline Hypoalbuminemia & 2.672 & $(I .634,3.7 I)$ & $<0.001$ & 1.479 & $(0.3,2.658)$ & 0.014 \\
\hline Lymphocytopenia & 0.289 & $(-0.79,1.367)$ & 0.599 & NA & NA & NA \\
\hline CRP, mg/L & 0.035 & $(0.021,0.049)$ & $<0.001$ & 0.021 & $(0.005,0.036)$ & 0.010 \\
\hline
\end{tabular}

Abbreviations: COVID-19, coronavirus disease; Cl, confidence interval; CRP, C-reactive protein; NA, not available.

regression analysis suggested that older age $(\beta-0.031$ [95\% CI, $-0.058,-0.004] ; \mathrm{P}=0.024)$ and increased CRP $(\beta-0.009[95 \% \mathrm{CI},-0.016,-0.001] ; \mathrm{P}=0.02)$ were negatively correlated with the number of days between the patient's appearance of hypoalbuminemia and hospital admission (Table 8).

\section{Changes of Albumin and Other Laboratory Tests in Patients Before and After Enteral Nutrition Therapy}

After enteral nutrition therapy, albumin (27.27 vs 41.38 , $\mathrm{P}<0.001$ ), leukocyte (17.53 vs 7.93, $\mathrm{P}<0.001)$, lymphocyte absolute value ( 0.47 vs $1.58, \mathrm{P}=0.003$ ), eosinophilic absolute value (0.04 vs $0.48, \mathrm{P}=0.007)$, CRP (202.7 vs $38.8, \mathrm{P}<0.001)$, and LDH (27.27 vs $41.38, \mathrm{P}<0.001)$ were all improved, and the differences were statistically significant (Figure 1).

\section{Discussion}

In this multicentre, retrospective study, we retrospectively analyzed 482 COVID-19 patients with a median age of 56 (IQR 39-67), 53.7\% of whom had decreased albumin over the course of the disease with a median albumin of $30.8 \mathrm{~g} /$ L (IQR 28.5-32.9). There may be several explanations for the inverse relationship between hypoalbuminemia and severe disease. As an anti-inflammatory and antioxidant 
Table 8 Linear Regression Analysis of Days Between the Appearance of Hypoalbuminemia and Hospital Admission in Patients with COVID-19 and Hypoalbuminemia

\begin{tabular}{|c|c|c|c|c|c|c|}
\hline \multirow{2}{*}{ Indicators } & \multicolumn{2}{|c|}{ Univariate Linear Regression } & \multirow[t]{2}{*}{$\mathbf{P}$} & \multicolumn{2}{|c|}{ Multiple Linear Regression } & \multirow[t]{2}{*}{$\mathbf{P}$} \\
\hline & $\boldsymbol{\beta}$ & $95 \% \mathrm{Cl}$ & & $\boldsymbol{\beta}$ & $95 \% \mathrm{Cl}$ & \\
\hline Age, years & -0.039 & $(-0.066,-0.012)$ & 0.005 & -0.031 & $(-0.058,-0.004)$ & 0.024 \\
\hline Dyspnea & -1.182 & $(-2.127,-0.237)$ & 0.014 & -0.716 & $(-1.659,0.226)$ & 0.136 \\
\hline C-reactive protein, $\mathrm{mg} / \mathrm{L}$ & -0.014 & $(-0.02,-0.007)$ & 0 & -0.009 & $(-0.016,-0.001)$ & 0.02 \\
\hline WBC count, $\times 10^{9} / \mathrm{L}$ & -0.17 & $(-0.27,-0.069)$ & 0.001 & -0.085 & $(-0.195,0.026)$ & 0.133 \\
\hline
\end{tabular}

Abbreviations: COVID-19, coronavirus disease 2019; Cl, confidence interval; CRP, C-reactive protein; WBC, white blood cell.

protein, albumin potentially protects against cytokine storms and preventing against multi-organ damage. Besides, the albumin contains the anticoagulant properties and inhibits coagulation and platelet activation associated with oxidative stress. ${ }^{7}$ Albumin is synthesized in the liver and has a serum half-life of approximately 21 days. ${ }^{8}$ Albumin is the most abundant plasma protein and plays an important role in maintaining blood colloid osmolarity, metabolite transport and nutrition in the body, which can reflect the nutritional status of patients and is a common laboratory test for hospitalized patients. Serum albumin levels are associated with the prognosis of pneumonia, severe sepsis, and bacteremia., ${ }^{4,9}$ Our findings of an increased risk of hypoalbuminemia in patients with severe
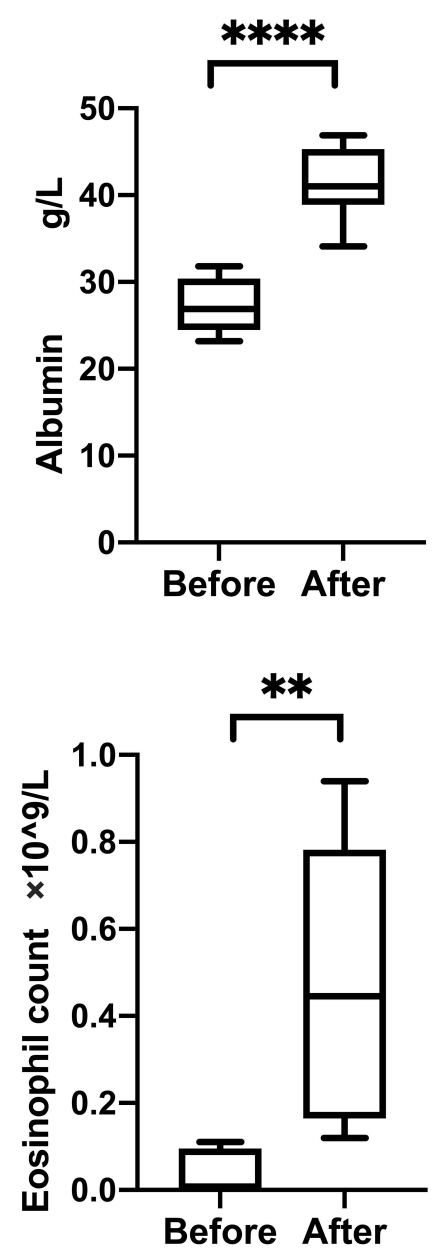
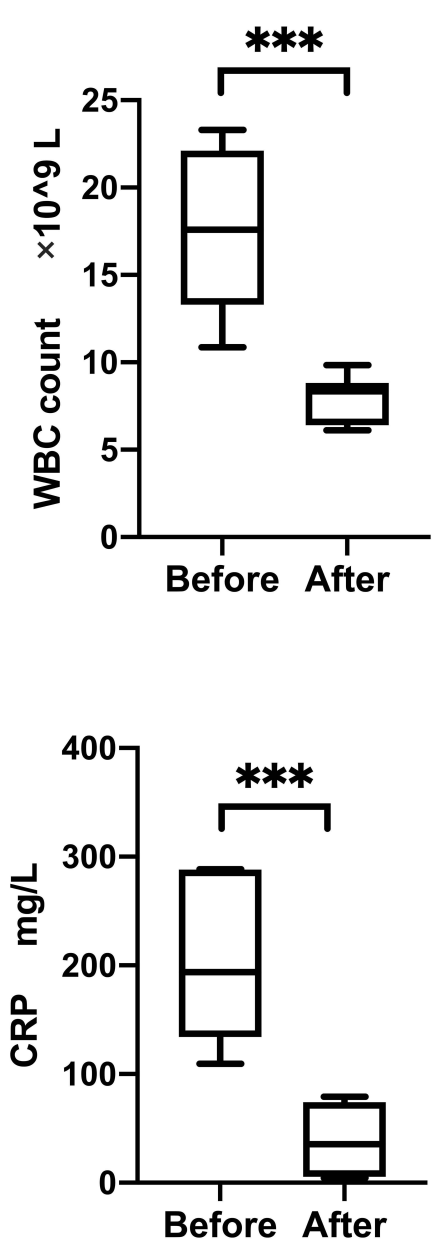
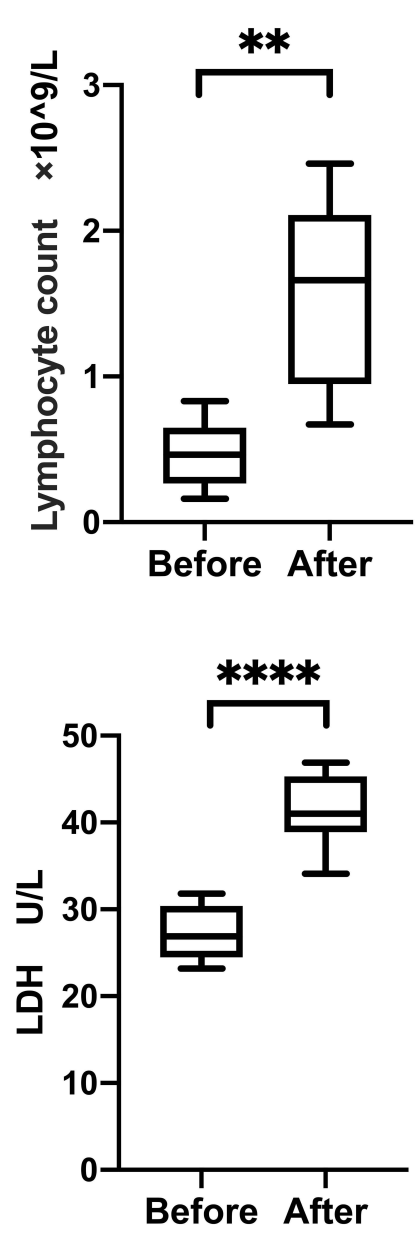

Figure I Changes in albumin and other laboratory tests before and after enteral nutrition therapy in 10 patients with COVID-20I9 and hypoalbuminemia. Statistical analysis was achieved by the $t$-test. $* * \mathrm{P}<0.01$, $* * * \mathrm{P}<0.001$ and $* * * * \mathrm{P}<0.0001$.

Abbreviations: COVID-19, coronavirus disease 2019; WBC, White blood cell; CRP, C-reactive protein; LDH, Lactate dehydrogenase. 
COVID-19 were consistent with previous reports. Lower serum albumin can be indicative of malnutrition, underlying disease, or infectious processes. ${ }^{10}$ Albumin was also regarded as a reliable indicator of the prognosis of patients with severe COVID. ${ }^{11}$ Hypoalbuminemia is common in SARS patients, and inversion of the $\mathrm{A} / \mathrm{G}$ ratio can indicate SARS patients earlier. ${ }^{12,13}$ Hypoalbuminemia negatively affects the immune response system and increases shortterm mortality in hospitalized stroke patients with acute infectious diseases. ${ }^{14}$ For each unit increase in serum albumin, the chance of death reduced $14 \% .^{15,16}$ Patients with concomitant hypoalbuminemia have higher mortality rates and longer hospital stays. ${ }^{12}$ In a recent study on COVID-19 patients, hypoalbuminemia was found to be an independent risk factor for death, and the risk of death in patients with hypoalbuminemia was 6.394 times higher than that in patients with normal albumin. ${ }^{17}$ In our study, we found that the mortality rate of patients with hypoalbuminemia was significantly higher than that of patients with normal albumin $(23.85 \%$ vs $0.9 \%)$, and the proportions of patients with normal lung CT, normal CRP and normal lymphocytes at discharge were lower than that of patients with normal serum albumin levels. We also found that COVID-19 patients with hypoalbuminemia were 2.121 times more likely to have severe disease and 1.479 times more likely to be hospitalized than patients with normal albumin, indicating that patients with hypoalbuminemia were sicker, had a poorer prognosis, and required a longer period of treatment.

Our study showed that in COVID-19 patients, age was an independent predictor of decreased albumin, with a $4.4 \%$ increase in the risk of hypoalbuminemia for each additional year of age. Previous studies have shown that serum albumin concentrations decreasing by $0.08-0.17 \mathrm{~g} /$ $\mathrm{L}$ per year, more in men than in women, ${ }^{18,19}$ and more markedly in the presence of sepsis, poor liver function and severe disease. ${ }^{20}$ Decreased albumin in the elderly may be related to their reduced dietary intake due to changes in taste and smell, changes in hormones that regulate gastrointestinal motility, and changes in mood. ${ }^{21}$ Secondly, the ability of the liver to synthesize albumin decreases with increasing age. However, it has also been reported that albumin concentrations do not decrease with age, ${ }^{22}$ and these inconsistencies may be related to the state of the organism or the individual's lifestyle by altering the rate of synthesis, hepatocyte secretion, humoral fluid distribution, degradation rate, and the degree of exogenous loss. ${ }^{23}$
CRP and lymphocyte counts were important indicators of systemic inflammatory response in clinical practice.In our study, increased CRP and decreased lymphocyte count were independent predictors of hypoalbuminemia, which corroborated the study of Mo, Zhang et al. ${ }^{24,25}$ Each $1 \mathrm{mg} / \mathrm{L}$ increase in CRP increases the risk of hypoalbuminemia in COVID-19 patients by $3.8 \%$. In response to tissue damage and inflammation, serum CRP levels rise rapidly and activate the body's defense mechanisms rapidly. ${ }^{26}$ Each $0.1 \mathrm{~g} / \mathrm{L}$ decrease in lymphocytes increases the risk of hypoalbuminemia in COVID-19 patients by $4.5 \%$. Lymphocyte is an important cellular component of the body's immune response function, and a decrease in lymphocyte indicate that a patient is in the acute phase of certain infectious diseases. ${ }^{27}$ It has been shown that elevated CRP levels and decreased lymphocytes were poor predictors of pneumonia progression and respiratory failure in MARS patients and that initial CRP levels were associated with prognosis in SARS patients. ${ }^{15,16}$ In COVID-19, CRP levels and lymphocyte counts were also found to correlate with disease severity. ${ }^{2}$ The systemic inflammatory response results in increased vascular permeability and extracellular migration of albumin, which may be responsible for the decrease in albumin. Whether the prognosis of patients could be assessed in combination with age, symptoms, albumin, CRP, lymphocyte count, and liver function status needs to be further investigated. In addition, we found that the older the patient and the higher the CRP, the shorter the time between admission and the appearance of hypoalbuminemia, which further indicated that age and CRP were associated with hypoalbuminemia in covid-19 patients.

At present, there is controversy over whether patients with low albumin need to be supplemented with human albumin. Current research is increasingly inclined to believe that hypoalbuminemia is only a manifestation of a disease rather than the cause, so simply supplementing albumin will not have much effect. There are strict indications for the intravenous use of albumin. Among the recommendations for the use of albumin and immunoglobulin, there is no recommendation for supplementing albumin in patients with severe pneumonia. ${ }^{28}$ The treatment of hypoalbuminemia is mostly aimed at the treatment of protopathy and enteral/parenteral nutrition support.,29 After enteral nutrition therapy administered to 10 COVID-19 patients with hypoalbuminemia, their albumin levels were corrected, leukocytes, lymphocytes, eosinophils, CRP and LDH were restored at the same time, 
which proved that correcting the serum albumin levels of COVID-19 patients with hypoalbuminemia could alleviate the systemic inflammation of the patients.

Our study also has shortcomings. First, this study was retrospective, and the medical history of some cases was not sufficiently detailed to determine the patients' medications and changes in various parameters before hospital admission. Second, the direct evidence that SARS-CoV-2 causes hypoalbuminemia has not been confirmed, and more studies are needed to explore its pathogenesis. Also, because of the lowest values of albumin we selected during hospital admission for correlation analysis and outcome prediction, we did not reflect the dynamic changes in albumin levels during the evolution of COVID-19 disease. The correlation of albumin's dynamic changes with various factors and their predictive value deserved further exploration. And in cases where albumin was corrected, the causal relationship between albumin correction and improvement in other indicators could not be reflected.

\section{Conclusion}

In conclusion, our study showed that hypoalbuminemia was common in patients with COVID-19 and may be the result of a combination of malnutrition and inflammation in patients. COVID-19 patients with hypoalbuminemia tend to have more severe clinical manifestations and more abnormal biochemical tests, which may result in poorer clinical outcomes. Hypoalbuminemia could indicate a poorer prognosis and the necessity for more diverse treatments. Nutritional support therapy may improve the clinical outcome of these patients. The specific association of the degree of albumin reduction with the severity of COVID-19 and the prognosis of patients still needs to be further investigated.

\section{Abbreviations}

COVID-19, coronavirus disease 2019; SARS, severe acute respiratory syndrome; PCR, polymerase chain reaction; ESLD, end-stage liver disease; CRP, C-reactive protein; $\mathrm{IQR}$, interquartile range; $\mathrm{LDH}$, lactate dehydrogenase; OR, odds ratio; CI, confidence interval; WBC, white blood cell; AST, aspartate aminotransferase; ALT, alanine aminotransferase; GGT, glutamyl transpeptidase.

\section{Ethics Approval}

The study was approved by the Ethics Commission of Wuhan Tongji Hospital, Union Hospital, and Jin Yin-tan Hospital. All data were anonymized to maintain participants' privacy, and the study was conducted in accordance with the Declaration of Helsinki. In light of the retrospective and anonymous nature of the study, the Ethics Committee did not require written informed consent provided by participants.

\section{Acknowledgment}

The National Natural Science Foundation of China [grant number 82070572, 81770554, 81570501] supported this study. We also thank all medical workers for participating in diagnosis and treatment for patients in Wuhan and all the patients involved in this study.

\section{Author Contributions}

All authors made a significant contribution to the work reported, whether that is in the conception, study design, execution, acquisition of data, analysis and interpretation, or in all these areas; took part in drafting, revising or critically reviewing the article; gave final approval of the version to be published; have agreed on the journal to which the article has been submitted; and agree to be accountable for all aspects of the work.

\section{Disclosure}

Chaoyue Chen and Ying Zhang are co-first authors for this study. The authors declare no conflicts of interest in this work.

\section{References}

1. Adhikari SP, Meng S, Wu YJ, et al. Epidemiology, causes, clinical manifestation and diagnosis, prevention and control of coronavirus disease (COVID-19) during the early outbreak period: a scoping review. Infect Dis Poverty. 2020;9(1):29. doi:10.1186/s40249-02000646-x

2. Zhang J, Wang X, Jia X, et al. Risk factors for disease severity, unimprovement, and mortality in COVID-19 patients in Wuhan, China. Clin Microbiol Infect. 2020;26(6):767-772. doi:10.1016/j. cmi.2020.04.012

3. Abdeen Y, Kaako A, Ahmad Amin Z, et al. The prognostic effect of serum albumin level on outcomes of hospitalized COVID-19 patients. Crit Care Res Pract. 2021;2021. doi:10.1155/2021/9963274

4. Gatta A, Verardo A, Bolognesi M. Hypoalbuminemia. Intern Emerg Med. 2012;7(Suppl 3):S193-S199. doi:10.1007/s11739-012-0802-0

5. Michelis R, Sela S, Zeitun T, Geron R, Kristal B. Unexpected normal colloid osmotic pressure in clinical states with low serum albumin. PLoS One. 2016;11(7):e0159839. doi:10.1371/journal.pone.0159839

6. National Health Commission of the People's Republic of China; Diagnosis and treatment program of new coronary pneumonia (the seventh edition). Infect Dis Inform. 2020;33(1)1-6. doi:10.3969/j. issn.1007-8134.2020.01.001

7. Basili S, Carnevale R, Nocella C, et al. Serum albumin is inversely associated with portal vein thrombosis in cirrhosis. Hepatol Commun. 2019;3(4):504-512. doi:10.1002/hep4.1317

8. Rothschild MA, Oratz M, Schreiber SS. Serum albumin. Hepatology. 1988;8(2):385-401. doi:10.1002/hep.1840080234 
9. Feldman C, Kallenbach JM, Levy H, et al. Community-acquired pneumonia of diverse aetiology: prognostic features in patients admitted to an intensive care unit and a "severity of illness" core. Intensive Care Med. 1989;15(5):302-307. doi:10.1007/BF00263865

10. Allard L, Ouedraogo E, Molleville J, et al. Malnutrition: percentage and association with prognosis in patients hospitalized for coronavirus disease 2019. Nutrients. 2020;12(12):E3679. doi:10.3390/nu12123679

11. Qin S, Li W, Shi X, et al. 3044 Cases reveal important prognosis signatures of COVID-19 patients. Comput Struct Biotechnol J. 2021;19:1163-1175. doi:10.1016/j.csbj.2021.01.042

12. Franch-Arcas G. The meaning of hypoalbuminaemia in clinical practice. Clin Nutr. 2001;20(3):265-269. doi:10.1054/clnu.2001.0438

13. Aziz M, Fatima R, Lee-Smith W, Assaly R. The association of low serum albumin level with severe COVID-19: a systematic review and meta-analysis. Crit Care. 2020;24(1):255. doi:10.1186/s13054-02002995-3

14. Dziedzic T, Pera J, Klimkowicz A, et al. Serum albumin level and nosocomial pneumonia in stroke patients. Eur $J$ Neurol. 2006;13 (3):299-301. doi:10.1111/j.1468-1331.2006.01210.x

15. Wang JT, Sheng WH, Fang CT, et al. Clinical manifestations, laboratory findings, and treatment outcomes of SARS patients. Emerg Infect Dis. 2004;10(5):818-824. doi:10.3201/eid1005.030640

16. Ko JH, Park GE, Lee JY, et al. Predictive factors for pneumonia development and progression to respiratory failure in MERS-CoV infected patients. J Infect. 2016;73(5):468-475. doi:10.1016/j.jinf.2016.08.005

17. Huang J, Cheng A, Kumar R, et al. Hypoalbuminemia predicts the outcome of COVID-19 independent of age and co-morbidity. $\mathrm{J} \mathrm{Med}$ Virol. 2020;92(10):2152-2158. doi:10.1002/jmv.26003

18. Cabrerizo S, Cuadras D, Gomez-Busto F, Artaza-Artabe I, Marín-Ciancas F, Malafarina V. Serum albumin and health in older people: review and meta analysis. Maturitas. 2015;81(1):17-27. doi:10.1016/j.maturitas.2015.02.009

19. Gom I, Fukushima H, Shiraki M, et al. Relationship between serum albumin level and aging in community-dwelling self-supported elderly population. $J$ Nutr Sci Vitaminol. 2007;53(1):37-42. doi: $10.3177 /$ jnsv. 53.37
20. Giovannini I, Chiarla C, Giuliante F, Vellone M, Ardito F, Nuzzo G. The relationship between albumin, other plasma proteins and variables, and age in the acute phase response after liver resection in man. Amino Acids. 2006;31(4):463-469. doi:10.1007/s00726-0050287-5

21. Malafarina V, Uriz-Otano F, Gil-Guerrero L, Iniesta R. The anorexia of ageing: physiopathology, prevalence, associated comorbidity and mortality. A systematic review. Maturitas. 2013;74(4):293-302. doi:10.1016/j.maturitas.2013.01.016

22. Fu A, Nair KS. Age effect on fibrinogen and albumin synthesis in humans. Am J Physiol. 1998;275(6):E1023-E1030. doi:10.1152/ ajpendo.1998.275.6.E1023

23. Thalacker-Mercer AE, Johnson CA, Yarasheski KE, Carnell NS, Campbell WW. Nutrient ingestion, protein intake, and sex, but not age, affect the albumin synthesis rate in humans. $J$ Nutr. 2007;137 (7):1734-1740. doi:10.1093/jn/137.7.1734

24. Mo P, Xing Y, Xiao Y, et al. Clinical characteristics of refractory COVID-19 pneumonia in Wuhan, China. Clin Infect Dis. 2020; ciaa270. doi:10.1093/cid/ciaa270

25. Zhang Y, Zheng L, Liu L, Zhao M, Xiao J, Zhao Q. Liver impairment in COVID-19 patients: a retrospective analysis of 115 cases from a single centre in Wuhan city, China. Liver Int. 2020;40 (9):2095-2103. doi:10.1111/liv.14455

26. Sproston NR, Ashworth JJ. Role of C-reactive protein at sites of inflammation and infection. Front Immunol. 2018;9:754. doi:10.3389/fimmu.2018.00754

27. Berthelot JM, Lioté F, Maugars Y, Sibilia J. Lymphocyte changes in severe COVID-19: delayed over-activation of STING? Front Immunol. 2020;11:607069. doi:10.3389/fimmu.2020.607069

28. Liumbruno GM, Bennardello F, Lattanzio A, Piccoli P, Rossettias G. Recommendations for the use of albumin and immunoglobulins. Blood Transfus. 2009;7(3):216-234. doi:10.2450/2009.0094-09

29. Allison SP, Lobo DN, Stanga Z. The treatment of hypoalbuminaemia. Clin Nutr. 2001;20(3):275-279. doi:10.1054/clnu.2001.0440
Infection and Drug Resistance

\section{Publish your work in this journal}

Infection and Drug Resistance is an international, peer-reviewed openaccess journal that focuses on the optimal treatment of infection (bacterial, fungal and viral) and the development and institution of preventive strategies to minimize the development and spread of resistance. The journal is specifically concerned with the epidemiology of antibiotic resistance and the mechanisms of resistance development and diffusion in both hospitals and the community. The manuscript management system is completely online and includes a very quick and fair peerreview system, which is all easy to use. Visit http://www.dovepress.com/ testimonials.php to read real quotes from published authors. 